\title{
Social science students' concept images and concept definitions of anti-derivatives
}

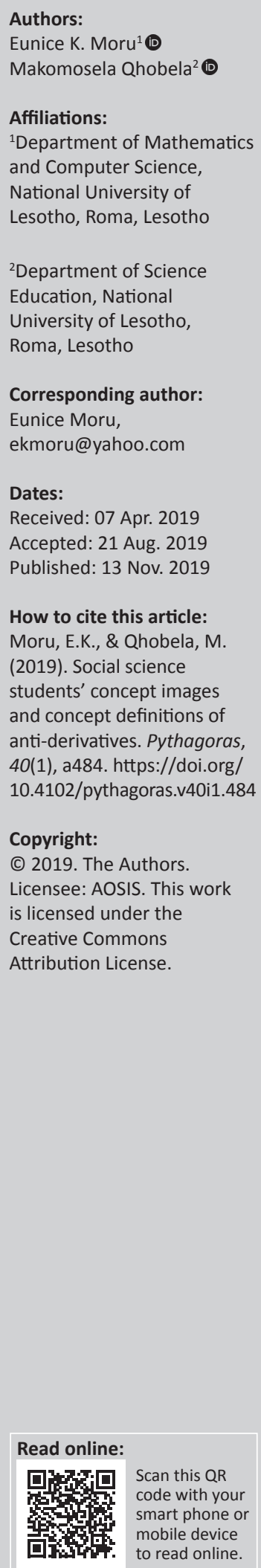

The purpose of the reported study was to investigate the social science students' concept images and concept definitions of anti-derivatives. Data were collected through asking students to answer 10 questions related to anti-derivatives and also by interviewing them. The theory of concept image and concept definition was used for data analysis. The results of the study show that the students' definitions of anti-derivatives were personal reconstructions of the formal definition. Their concept images were coherent only to a certain extent as there were some conceptions of some ideas that were at variance with those of the mathematical community. These were more evident when students solved problems in the algebraic representation. Some students did not know which integration or differentiation methods they should apply in solving the problems. The significance of such findings is to enable the mathematics educators to pay attention not only to the use of signs and symbols representing mathematical concepts but also to their semantics.

Keywords: anti-derivatives; concept definition; concept image; social science students; integration.

\section{Introduction}

Calculus is an important branch of mathematics in a number of social and natural science disciplines. In the social sciences anti-derivatives are required in tackling problems in marginal analysis and optimisation problems. In the natural sciences students need to understand antiderivatives to deal with rates of change for concepts such as velocity, acceleration and rate of flow. However, students fail to understand some of the basic concepts of calculus (Brijlall \& Ndlazi, 2019; Maharaj, 2014; Metaxas, 2007; Grundmeier, Hansen \& Sousa, 2006). For example, students have problems in relating fundamental concepts and their definitions with associated procedures. This disconnect suggests that students have difficulties understanding functional and operational relationships between the mathematical notations and the calculus concepts they represent. What is desirable is that mathematical procedures be understood conceptually, but this is not necessarily the case since many students continue to learn mathematical procedures by rote application of an algorithm (Moru \& Qhobela, 2013). A general anti-derivative, which is sometimes referred to as an indefinite integral, is an essential concept in calculus because it serves as a basis for many reallife problems and when students continue further into calculus courses, they frequently encounter anti-derivatives more than derivatives (Jones, 2013).

Like other calculus concepts the learning and understanding of anti-derivatives involves significant use of signs and symbols. But these symbols continue to lack meaning for students (Ferrer, 2016; Sengul \& Katranci, 2015). To accomplish proper understanding of this concept various researchers have tried different ways to improve its teaching and learning. Tall and Vinner (1981) have suggested investigating students' concept images and concept definitions of mathematical concepts. As they suggest, understanding a mathematical concept involves forming the concept image of it. Brijlall and Ndlazi (2019) have suggested developing the genetic decomposition of a concept (that is suggesting ways of how the concept might be learnt) so that instructors may organise their teaching of the concept for learning to take such a route. The current study was therefore conducted with the purpose of documenting university students' concept images and concept definitions of anti-derivatives, an important idea, applicable in the social sciences. This was with the intention to find ways to improve the teaching of the concept. We believe that one cannot remedy what they have not diagnosed. In the mathematical context the terms anti-derivative and indefinite integral may be used interchangeably; in this study the choice of using anti-derivative as opposed to indefinite integral is because everyday etymology suggests that the prefix 'anti' in the term 'anti-derivatives' implies the reverse process and product of derivatives. This interpretation poses problems in the mathematical context because our 
everyday meaning of 'reverse' means going back to where one started. Thus this would show if students would use the everyday meaning or the scientific meaning where the reverse processes of integration and differentiation may be used depending on the task at hand and their products would be either integrals or derivatives. The other reason for this choice was because the term 'derivative' has a close relationship with the word 'anti-derivative' as it constitutes the part of it after the prefix 'anti'.

Having highlighted some difficulties that students have in relation to the topic of study, studies related to students' understanding of this important calculus concept have not yet been conducted in Lesotho, where the current study took place. This could probably be because in Lesotho students encounter calculus for the first time at university or other tertiary institutions, of which there are not many; hence, the idea is not very popular. The findings of this study will not only be useful to the National University of Lesotho where the study was conducted, they will also be useful to researchers elsewhere in terms of either similarities or differences in findings and their implications.

This study addressed the following research questions:

- Research question 1 (RQ1): What definitions of antiderivatives do second-year social sciences students give?

- Research question 2(RQ2): What connections do students make between a function and its anti-derivative in algebraic representation?

- Research question 3 (RQ3): How do students solve problems involving differentiation and integration?

The foundation of this study involved the characteristics of a specific calculus concept, the anti-derivative. The theoretical framework by Tall and Vinner (1981) on concept image and concept definition was the main lens through which the students' understanding of the concept was analysed. This theory was complemented by some parts of the literature review.

\section{Literature review}

The foundation of this study involved characteristics and understanding of a very specific calculus concept. The review of the literature includes the type of difficulties that the students encounter in understanding the concept of antiderivative together with possible causes for such.

\section{Students' difficulties in understanding anti- derivatives (or indefinite integral).}

In the studies of Hall (2010), and Pino-Fan, Gordillo, Font, Larios and Castro (2017), students were explicitly asked to define an indefinite integral and an anti-derivative, whereas in the study by Metaxas (2007) students were provided with a set of words that they could use to describe the indefinite integral. The words that were provided for the description were: object, process, tool and concept. Hall (2010) found that everyday language affected the students' understanding of the word 'indefinite' in indefinite integral with the everyday meaning being 'less precise', a meaning that is not correct in the mathematical context. When asked what an indefinite integral is, a student gave the following response: 'an indefinite integral, I mean, it's more ... it's more open ended, less precise, obviously' (Hall, 2010, p. 12). The student is aware that in finding an indefinite integral, the limits of integration are not used. The problem is that in this case instead of using the correct mathematical explanation, inappropriate terminology that is non-mathematical is used. In the study of Pino-Fan et al. (2017) some students described an anti-derivative as 'the inverse of derivation' and other described it as a procedure that allows one to find the 'original function' from which a derived function comes (p. 41).

Metaxas's (2007) exploration of students' classification of their understanding of indefinite integral was in the context in which students had been introduced to the definition of an indefinite integral as: 'Indefinite integral of a function $f$ continuous in an interval $[a, b]$, is defined to be the set of all the anti-derivatives of $f$ in $[a, b]^{\prime}$ (p. 266). The student who was interviewed said that an indefinite integral is a process and this was interpreted as a conception that shows procedural understanding. This is a reflection that in both cases students had their own personal reconstruction of what an indefinite integral is.

The definition provided in the study of Metaxas (2007) implies that one indefinite integral (a general anti-derivative) contains a number of particular anti-derivatives. Students in the Metaxas study were also asked if $\int \frac{1}{x} d x$ is equal to. $1+\int \frac{1}{x} d x$ During the interview one student said that the two indefinite integrals are not equal. The student's interpretation seems to be in alignment with the idea of initial value problems where the constants of particular antiderivatives of the two functions would differ by 1 (one). If written in the general form the answer would be given as $\int \frac{1}{x} d x=1+\int \frac{1}{x} d x=\ln |x|+c$.

On the surface the two integrals seem to produce the same function but in essence the $c$ stands for an infinite number of constants which may not necessarily be equal.

In the study by Kiat (2005), when students were asked to find $\int \cos (2 x-1) d x$ some students gave the answer as $\frac{1}{2} \sin (2 x-1)$ instead of $\frac{1}{2} \sin (2 x-1)+c$. Thus, by omitting a constant of integration, a procedural error was committed. In our view, it could be that students were not aware that an integral (a general anti-derivative) is constituted by a set of particular antiderivatives as the $c$ stands for varying constants. Thus, $\frac{1}{2} \sin (2 x-1)$ is one of the anti-derivatives with $c=0$ and writing it down alone ignores the other anti-derivatives with different constants. On the other hand there is a possibility that the students just forgot to write the $c$ especially when a procedure was just learned as a set of rules without meaning attached.

Giving incorrect answers due to calculation errors, failure to use relevant rules and to link derivatives to their 
anti-derivatives and inability to use appropriate integration techniques in solving indefinite integral problems by students were dominant in the studies by Maharaj (2014), Sengul and Katranci (2015), Ferrer (2016), Brijlall and Ndlazi (2019), Ndlazi and Brijlall (2019). In the study by Maharaj students made some structural errors as they could not differentiate objects from processes from the way the integrals were represented. A process was defined as the ability to perform operations that will enable the student to find the integrals of functions while an object was taken as the visualisation of strings of processes as a totality and performing some written or mental actions on the internal structure of the given function to enable integration. In Sengul and Katrachi, the choice of strategy to use was a serious problem. Ferrer asserts that learners' difficulties in solving integrals were attributed to the inherent mathematical knowledge and skills acquired by the students from basic mathematics. In Brijlall and Ndlazi's studies, students committed errors when using symbols of integration and basic differentiation rules. Students also had problems with the syntax of symbols as in the case of Maharaj.

\section{Theoretical framework}

The theory of concept definition and concept image by Tall and Vinner (1981) and Vinner (1991) was used for data analysis. This theory was complemented by parts of the literature that were directly related to the concept of study. These include: the formal definition of anti-derivative, its relationship with indefinite integral, and the relationship between differentiation and integration.

\section{Concept image and concept definition}

Tall and Vinner (1981) describe the formal concept definition as the form of words used to specify the concept as accepted by the mathematical community. They suggest that this may be learnt by an individual in a rote fashion or more meaningfully learned and related to a greater or lesser degree to the concept as a whole. It may also be a personal reconstruction of the definition by the student which may differ from the formal concept definition.

The term concept image is described as the total cognitive structure that is associated with the concept, which includes all the mental pictures and associated properties and processes (Tall \& Vinner, 1981, p. 152). Part of the concept image that is activated at a particular time is termed the evoked concept image. At different times conflicting images may be evoked. Cognitive conflict or confusion occurs when conflicting aspects are evoked simultaneously. Vinner (1991) contends that to know a concept definition by heart does not guarantee understanding of the concept. He says that to understand a concept means to have a concept image of it.

The definitions of anti-derivative provided in some calculus textbooks include a list of connected conditions: 'An antiderivative of a function $f$ is a function $F$ such that $F^{\prime}(x)=f(x)^{\prime}$ (Haeussler, Paul \& Wood, 2008, p. 624); 'A function $F$ is called an anti-derivative of $f$ on an interval $I$ if $F^{\prime}(x)=f(x)$ for all $x$ in $I^{\prime}$ (Stewart, 2009, p. 274); 'A function $F$ is an anti-derivative of $f$ on an interval $I$ if $F^{\prime}(x)=f(x)$ for all $x$ in $I^{\prime}$ (Larson \& Edwards, 2012, p. 398). These authors go on further to show that any two anti-derivatives (particular) of a function differ only by a constant and that an indefinite integral is the most general anti-derivative. Larson and Edwards (2012, p. 399) state that 'the term indefinite integral is a synonym for antiderivative' and to find a particular anti-derivative one has to solve the initial value problem.

These definitions mean that if we have a function $F$, it qualifies as an anti-derivative of another function $f$ if when differentiated it gives $f$ as the result. In order to check if $F$ is an anti-derivative of $f$, we simply have to differentiate $F$. If we get $f$, then we can conclude that $F$ is the anti-derivative of $f$. This further suggests implicitly that if we integrate $f$ we will get $F$, which is not necessarily the case. This implied mathematical meaning as a symbolic representation is $\frac{d}{d x}\left(\int f(x) d x\right)=f(x)$ and $\int \frac{d}{d x}(F(x)) d x=F(x)+c$, where $c$ is the constant of integration (Haeussler et al., 2008). Therefore, if we integrate (integration operator $\int$ ) a function $f$, then differentiate (differentiation operator $\frac{d}{d x}$ ), the result will be what we started with. However, if we differentiate $F$ and then integrate, we will not necessarily go back to exactly where we started because $F(x)+c$ is an expression for a family of functions rather than the single function, $F(x)$, that we started with. Although the researchers agreed with all the given definitions, the students were exposed to the definition by Haeussler et al. (2008); hence, that is the one that the sample of the study was expected to use.

\section{Methodology Research design}

This study involved two methods of data collection to document students' understanding of anti-derivatives. Questions on anti-derivatives and interviews to illuminate the students' questions responses were used. These methods of data collection were important because they could both enable the researchers to get qualitative data that could answer the research questions by studying students' responses to get direct answers or make inferences.

\section{The sample}

The participants in this study were 117 second-year university social science students whose major subjects were economics and statistics. The sample group was taught algebra and calculus in their first and second years by the first author. The calculus topics covered during their second year included: integrals, techniques of integration and the relationship between integration and differentiation with initial value problems.

Students were also aware that the indefinite integral was used as a general anti-derivative. The students had encountered problems in which the terms anti-derivative and integral (indefinite) had been used interchangeably in 
their calculus textbooks. They also had solved application problems such as: marginal cost, marginal revenue, marginal propensity to save, marginal propensity to consume, and initial value problems. Students knew for example that the cost function is the anti-derivative of the marginal cost function obtained through the process of integration and to check this they had to differentiate to obtain the marginal cost function. The students had not solved problems in which the integration and differentiation operators were paired. They encountered this for the first time during the study. They were also not familiar with the integration of expressions that cannot be broken down into elementary functions. The learning took place through lectures, class discussions and tutorial sessions. The participants had used mainly the prescribed calculus textbook in the social sciences (Haeussler et al., 2008), which was recommended on the basis of relevance to the course of study.

\section{The questions}

Ten questions (see Table 1) were constructed by both authors and validated by two mathematics educators from universities outside Lesotho. The comments from these experts were discussed to reach agreement and the consensus suggestions were used to revise the questionnaire.

\section{Interviews}

Interviews were used to ensure validity and provide richer elaborations of the questionnaire responses. This also provided a form of methodological triangulation (Seale, 1999). The semi-structured protocol was constructed by the authors and external assessors were involved until an agreement was reached. The first author conducted the interviews to maximise the reliability of the responses since she had already established a rapport with the students as their lecturer. The interviews were audio-taped to preserve

TABLE 1: The questions, their justification and research questions their data would answer.

\begin{tabular}{|c|c|c|}
\hline Question & Why it was asked & Research question that would be answered by its data \\
\hline 1. What is an anti-derivative of a function? & To find how students would define an anti-derivative. & $\begin{array}{l}\text { Data collection was useful in responding to Research } \\
\text { question } 1 \text { (RQ1). }\end{array}$ \\
\hline $\begin{array}{l}\text { 2. Does a function have only one anti-derivative? } \\
\text { Explain. }\end{array}$ & $\begin{array}{l}\text { To give students an opportunity to relate a function } \\
\text { to its anti-derivative. }\end{array}$ & $\begin{array}{l}\text { Data collected was useful in responding to Research } \\
\text { question } 2 \text { (RQ2). }\end{array}$ \\
\hline $\begin{array}{l}\text { 3. In order to complete the process of integration } \\
\text { a constant must be added to the results. Why is } \\
\text { this so? }\end{array}$ & $\begin{array}{l}\text { To give students an opportunity to relate a function } \\
\text { to the indefinite integral which is taken to be the } \\
\text { general anti-derivative of a function. }\end{array}$ & Data collected was useful in responding to RQ2. \\
\hline $\begin{array}{l}\text { 4. What is the relationship between the processes } \\
\text { of differentiation and integration if any? }\end{array}$ & $\begin{array}{l}\text { To find if students could relate the two processes } \\
\text { verbally so that they could use this knowledge to } \\
\text { solve problems that could not be broken down into } \\
\text { simpler functions when integrated. }\end{array}$ & $\begin{array}{l}\text { Data collected was useful in responding to Research } \\
\text { question } 3 \text { (RQ3). }\end{array}$ \\
\hline $\begin{array}{l}\text { 5. Find: } \\
\text { a. } \frac{d}{d x}\left(\frac{x^{n+1}}{n+1}+c\right) \\
\text { b. Which processes did you use in finding the } \\
\text { answer for } 5 \text { a? }\end{array}$ & $\begin{array}{l}\text { To find students' procedural understanding of } \\
\text { differentiation. }\end{array}$ & Data collected was useful in responding to RQ3. \\
\hline $\begin{array}{l}\text { 6. Find } \\
\text { a. } \int x^{n} d x \\
\text { b. Which process did you use in finding the } \\
\text { answer for } 6 a \text { ? }\end{array}$ & $\begin{array}{l}\text { To find students' procedural understanding of the } \\
\text { process of integration. }\end{array}$ & Data collected was useful in responding to RQ3. \\
\hline $\begin{array}{l}\text { 7. What is the relationship between the functions } \\
g(x) x^{n} \text { and } f(x)=\frac{x^{n+1}}{n+1}+c \text {, if any? }\end{array}$ & $\begin{array}{l}\text { To provide students an opportunity to relate the } \\
\text { general forms of functions where one is the derivative } \\
\text { and the other is the integral. }\end{array}$ & Data collected was useful in responding to RQ2. \\
\hline $\begin{array}{l}\text { 8. Find } \\
\text { a. } \frac{d}{d x} \int g(x) d x \\
\text { b. } \int \frac{d}{d x}(g(x)) d x\end{array}$ & $\begin{array}{l}\text { To see if students could relate the processes of } \\
\text { integration and differentiation in a general algebraic } \\
\text { form. }\end{array}$ & Data collected was useful in responding to RQ3. \\
\hline $\begin{array}{l}\text { 9. Find } \\
\text { a. } \int \frac{d}{d x}\left(x^{2}\right) d x \\
\text { b. } \int \frac{d}{d x}\left(e^{x^{2}}\right) d x \\
\text { c. } \frac{d}{d x} \int e^{x^{2}} d x\end{array}$ & $\begin{array}{l}\text { To see if students could relate the processes of } \\
\text { integration and differentiation in a general algebraic } \\
\text { form with functions that could not be broken down } \\
\text { into simpler forms. }\end{array}$ & Data collected was useful in responding to RQ3. \\
\hline $\begin{array}{l}\text { 10. Find } \\
\text { a. } \frac{d}{d x} \int 3 x^{3} d x \\
\text { b. } \int \frac{d}{d x} e^{x^{3}} d x \\
\text { c. } \frac{d}{d x} \int e^{x^{3}} d x\end{array}$ & $\begin{array}{l}\text { To see if students could relate the processes of } \\
\text { integration and differentiation in a general algebraic } \\
\text { form with functions that could not be broken down } \\
\text { into simpler forms. }\end{array}$ & Data collected was useful in responding to RQ3. \\
\hline
\end{tabular}


data in a 'raw' form in that it removes the selective effect of researchers (Seale, 1999).

The interviews were conducted with a stratified subsample of participants $(n=12)$. Four interviewees were chosen from each of the first two popular categories of responses and two from each of the two least popular categories of responses based on the analysis of the questions (see Table 2). Interview questions were aligned with and supplemented each category of response from the questionnaires. For example, the first interview question was constructed by considering the four categories of responses (an integral, integration, reverse and original function). The same procedure was applied to questions 1, 2, 3, 4 and 9. The other interview questions were asked to find out if the students could successfully solve problems involving the procedures used in finding anti-derivatives and their relationship with their description of what an anti-derivative is. On average each interview lasted one hour.

\section{Data analysis}

Data was analysed using the theory of concept image and concept definition. The theory was complemented by some parts of the literature. For example, in analysing the students' definition of anti-derivatives (see Table 2) students' answers were compared with the formal definition of the anti-derivative as accepted by the mathematical community which was already known to the authors beforehand (a priori knowledge). If the answers did not match the formal definition, they were taken to be students' personal definitions. In forming the classifications of the personal definitions the authors looked for the common words that were used by the students. Such words seemed to be related to the language that in most cases is used in the context of anti-derivatives. Such words include: reverse, integration, integral and original function. These personal definitions, although they contained familiar terms within the context of anti-derivatives, were only classified after reading students' responses (posteriori knowledge). The authors did not have any prior knowledge of how students would define anti-derivatives through their personal reconstructions of the formal definition they had encountered in class.
In analysing data that relates a function to its anti-derivative, two possibilities were anticipated by the authors: (1) that a function has one anti-derivative or (2) that a function has many anti-derivatives. These two categories were anticipated because there is a possibility that students could take the general anti-derivative as one function if they are not aware that the $c$ in the expression is an arbitrary constant. In analysing data for the third research question, students' definitions of anti-derivatives and the way they related the processes of integration and differentiation were used as a major lens. The given students' definitions were checked if they matched some of their explanations or the way they solved the given problems represented in algebraic form, thus checking students' concept images (the total cognitive structure) of anti-derivative. Comparison with results found by researchers elsewhere was also made.

\section{Ethical considerations}

Students were asked if they were willing to take part in the research by the first author who taught them. They were made aware that any information they give will be treated with great confidentiality. This is also evidenced by the fact that their names are not used in the research and they were aware of this. They were also told that they are free to withdraw from the research activity if they felt uncomfortable. But because of the good relationship that existed between the first author and the students, they showed great interest in taking part in the research. Perhaps another factor that contributed to students' willingness to participate is that they were told that the information that they give will also help their instructor to improve her teaching not only for their benefit but also for the classes that will follow. The permission to conduct the study was also obtained from the Head of Department of Mathematics and Computer Science as the course is offered by the mentioned department.

\section{Findings}

The presentation of results in this section is categorised into the assertions and evidence aligned with the research questions. The assertions are accompanied by supporting evidence composed of summaries of the questions' responses and specific in-depth quotes from the student interviews from different performance groups and discussion.

TABLE 2: Students' definitions of anti-derivative.

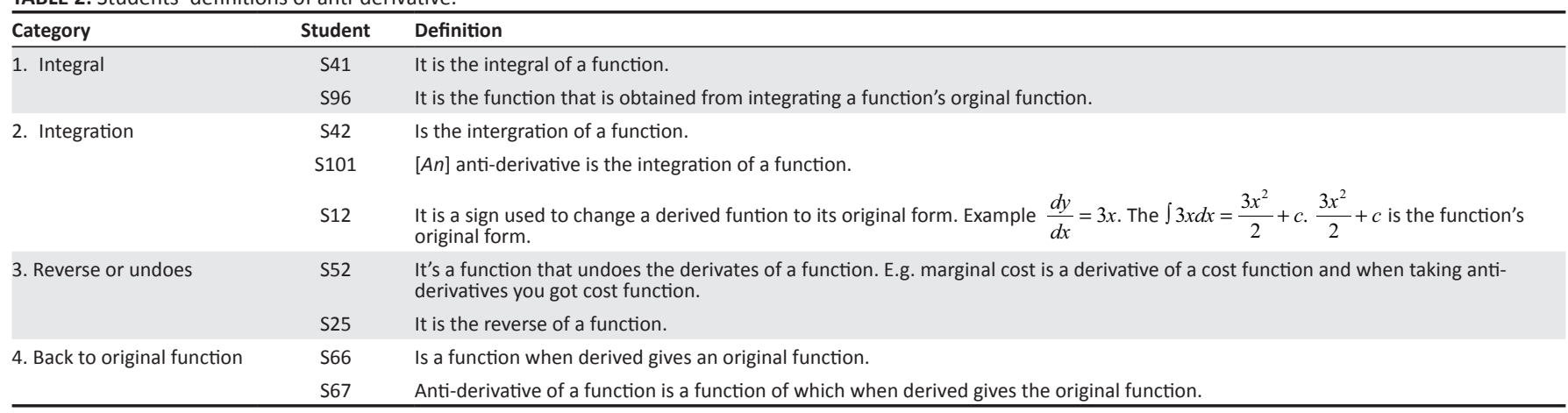




\section{RQ1 Assertion: Students provided four categories of definitions of anti-derivatives that are personal concept definitions}

From the 117 students' responses about the definition of antiderivative, 111 yielded four categories: an anti-derivative is the integral of a function (67 students $-60 \%$ of students including interviewees S41, S59, S96 and S100); an antiderivative is the integration of a function (34 students - 31\% of students including interviewees S12, S42, S48 and S101); an anti-derivative is the function that undoes (change, reverse) the derivative ( 7 students $-6 \%$ of students including interviewees S25 and S52); an anti-derivative is the function that when derived gives the original function (3 students $3 \%$ of students including interviewees S66 and S67). One student defined an anti-derivative as a function, there were no responses from two students and three students' responses were unclassified. Table 2 illustrates the actual responses of some students that were coded into the four categories.

The given definitions are students' own personal reconstruction of the formal concept definition; similar results were obtained in the studies of Metaxas (2007), Hall (2010) and Pino-Fan et al. (2017) when students were asked to give the definition of an indefinite integral (a general anti-derivative). In the first category, when students say that an anti-derivative is an integral it shows that they are aware that the two concepts are related. Integrals are either definite or indefinite and the latter is used synonymously with the term anti-derivative whereas the definite integral represents a number. The discussion with S41 from the first category went as follows.

$\mathrm{R}$ : What do you mean when you say that an anti-derivative is an integral of a function? What is an integral?

S41: The integral is the inverse of the derivative.

R: Can you show me what you mean by this.

S41: She then writes [talking through the steps]

$$
\begin{aligned}
& f(x)=2 x=2 \\
& \int 2 d x=2 x+c \\
& 2 \int x d x=\frac{2 x^{2}}{2} \\
& =\int x^{2}=\frac{x^{3}}{3}
\end{aligned}
$$

[As she writes she says:] If $f(x)$ is equal to $2 x$, then its derivative is 2 but the integration of 2 is going to be $2 x$.

R: Is it what you mean by inverse?

S41: Yes.

S41 associates the term anti-derivative with two terms, integral and inverse (of the derivative). We believe that this emanates from the use of the prefix 'anti' and also from the fact that differentiation and integration are referred to as reverse processes. In her working she starts by talking about the derivative, then shows how one can get the indefinite integral from the derivative. This shows her concept image of inverse which matches her personal concept definition. Her working shows that the process of integration was performed twice. Although the relationship between $2 x$ and 2 is shown and explained clearly, when integrating the integral $2 x$ the $c$ is left out and the second integration was not relevant as per the discussion.

In the second category, S42 and S101 use the term integration while S12 refers to the sign, an integration operator, which has the same fundamental meaning as integration. These showed that the students' concept images of anti-derivative had the process of integration as part of their cognitive structure. In the third category the words undoes and reverse are explicitly used in the definition. These words are commonly used when the two processes of integration and differentiation are discussed, whether in textbooks or in teaching. In the last category the students use the word derived for finding the derivative of a function. This category is aligned with the way one would check whether or not a function is an anti-derivative of another. If a function $\boldsymbol{F}$ is differentiated and the result is another function $f$, then $F$ is the anti-derivative of $f$.

In comparison with the findings of Hall (2010), where students' definitions of indefinite integrals were characterised by the use of everyday language, in this study that was not the case. In the current study students used words that are commonly associated with the description of indefinite integral, either explicitly in the explanations or implicitly in the formal definition when relating a function to its antiderivative. Thus the concept images of students in the reported study consist of mental structures that are more coherent in the mathematical sense than those displayed by students in the study of Hall. The same argument of coherence of definitions in the mathematical sense could be made for the study of Pino-Fan et al. (2017) as the categories of the definitions of students in their study are similar to categories 3 and 4 of the reported study: inverse (taken to be synonymous with reverse) and original form.

\section{RQ2 Assertion A: Students related a function to its anti-derivative with multiple interpretations}

The two questions that required students to explicitly relate a function to its anti-derivative are Question 2 and Question 7. Question 2 read: 'Does a function have one anti-derivative? Explain' while Question 7 read: 'What is the relationship between the functions $g(x)=x^{n}$ and $f(x)=\frac{x^{n+1}}{n+1}+c$, if any?' In responding to Question 2 the expectation was that the students would say that a function has many anti-derivatives, as taught in class. They were further expected to say that the function $f(x)=\frac{x^{n+1}}{n+1}+c$, is a set of anti-derivatives of
$g(x)=x^{n}$.

In responding to Question 2, 79 students (69\%), including S12, S25, S52, S59, S96 and S101, said that a function has many anti-derivatives. Of these respondents, 51 were aware that a function has many anti-derivatives that differ by a constant while 22 students, including S12 and S96, said that a function will have many anti-derivatives depending on the order of 
differentiation of the function. Six students did not explain their answers. Another 32 students (28\%), including interviewees, S42, S48, S66, S67 and S100, said that the function has only one anti-derivative, by which they meant a general anti-derivative. Four students (3\%) including interviewee $\mathrm{S} 41$ gave unique responses and two students did not respond. The response that a function has many antiderivatives does not mean that all the students had the same interpretation, as interview excerpts from S12 and S25 illustrate. Their questionnaire responses are first presented:

S12: It depends on how many times it has been derived. If it has been derived twice, then it will be anti-derived twice.

S25: No. It is because any anti-derivative has the constant $C$ which represents any number so function has many antiderivatives.

S12's interview:

$\mathrm{R}$ : When asked if a function has only one anti-derivative, you say that it depends on how many times it has been derived. Can you explain what you mean?

S12: I mean if a function has been derived twice as in $y^{\prime \prime}$, then you will integrate it twice to get to the original function $y$.

$\mathrm{R}$ : Can you explain what you mean.

S12: [To demonstrate what she means, she wrote:]

$y^{\prime \prime}=3 x^{2}$

$3 \int x^{2} d x$

$y^{\prime}=x^{3}+C$

$y=\int x^{3}$

$y=\frac{x^{4}}{4}$

[As she wrote she said:] If $y$ double prime is equal to $3 x$ squared, then you have to integrate. The answer will be $x$ cubed plus $c$ which is $y$ prime. To get $y$ you will have to integrate and if you are given the value of $c, y$ will be $x$ to the power four over four.

R: Can you show me on what you have written which functions are the anti-derivatives of the others?

S12: This integral is the derivative of $y$ double prime.

R: Which one?

S12: This ( $y$ prime) function is the anti-derivative of $y$ double prime.

$\mathrm{R}: \quad$ So the anti-derivative of $y$ double prime is $y$ prime, and that of $y$ prime is ...

S12: [Interrupting] It is $y$.

$\mathrm{R}: \quad$ Does this $c$ stand for any particular number?

S12: It stands for any particular number if we are not told how to find the $c$.

$\mathrm{R}: \quad$ Can it be $y^{\prime}=x^{3}+2$ or $y^{\prime}=x 3-3$ or $y^{\prime}=x^{3}-1$ ?

S12: Yes madam.

$\mathrm{R}: \quad$ So is $x^{3}+c$ one anti-derivative?

S12: Yes it is one.

S12's concept image shows that a function has many antiderivatives but these anti-derivatives are counted based on the number of times in which a function is integrated to its original form. The example she gives is that of a function which has two anti-derivatives because it has been integrated twice to get back to $y$. She accepts the existence of particular anti-derivatives but as she explains such anti-derivatives may exist only when one is provided with the conditions that will enable the calculation of $c$ which she takes to represent one number at a time. Her written work seems to indicate a problem in handling the $c$ when integrating. She does not manage to write its integral as $c x+k$, where $k$ is a constant. She seems to think that if you are given the value of $c$ the other part disappears or becomes zero as in the case of differentiating a constant. Thus she may confuse the processes of integration and differentiation, a situation which occurred in Kiat's (2005) study. S41 like S12 no longer refers to the antiderivative as the integral sign, integration operator, but as the integral. She also relates an anti-derivative with the last category, back to the original form. Thus the interviews further reveal that the categories of descriptions and antiderivatives overlap. S25 was among the students who said that a function had many anti-derivatives but his reasoning was different from that of S12 as indicated by his interview:

R: Can you explain what you mean when you say that a function has many anti-derivatives?

S25: Taking $x^{2}+\mathrm{c}$ as an example, c can be anything. Let's say we have $x^{2}+3, x^{2}+4$ and $x^{2}+k$, any constant; my understanding is that $x^{2}+3$ is different from $x^{2}+4$. Therefore, those antiderivatives are different.

$\mathrm{R}$ If you were to differentiate each of these anti-derivatives what would you get?

S25: They will be the same.

R: What will they be?

S25: They will be $2 x$.

R: Is this why you say a function has many anti-derivatives?

S25: Yes madam.

The excerpt shows that S25 gave the interpretation of anti-derivative as taught. Which also matches his personal concept definition of a reverse process. He takes an indefinite integral to be a set of anti-derivatives that differ by a constant as reflected by his questionnaire response. Thus his concept image of anti-derivative was coherent. The multiple interpretations of anti-derivatives given by students, though different, were the same for each student. This means that there were no conflicts that were experienced between the individual students' evoked concept images.

\section{RQ2 Assertion B: Students related a function to its anti-derivative the same way}

This is the second assertion made in trying to answer the second research question on how students related the function to its anti-derivative. S42 was among the students who said that a function has only one anti-derivative, the general, consistently. S12 also consistently related a function to its anti-derivative by saying that it depends on the number of times the function had been differentiated. S42's interview about her Question 2 response illustrates this point. S12's 
response to Question 2 has already been discussed and reference will be made to it when discussing both of their responses to Question 7.

S42: Yes. But it has different constants. $\int x^{n}=\frac{x^{n+1}}{n+1}+C$ (The only anti-derivative).

S42's interview:

$\mathrm{R}: \quad$ [Referring to earlier discussion] Can I say $g(x)=\frac{3 x^{2}}{2}+2$ is the anti-derivative of $f(x)=3 x$ ?

S42: No! $\mathrm{R}:$ If I have the integral $f(x)=\frac{3 x^{2}}{2}+c$, what can I do to this
integral in order to get $3 x$ ?

S42: We can differentiate.

$\mathrm{R}$ : What about the derivative of $w(x)=\frac{3 x^{2}}{2}+1$, what is it?

S42: It is $3 x$.

$\mathrm{R}: \quad$ Is $w(x)=\frac{3 x^{2}}{2}+1$ the anti-derivative of $3 x$ ?

S42: No! The anti-derivative is $\frac{3 x^{2}}{2}+c$.

S42's conception of anti-derivative is that of a general antiderivative. She said that $\frac{3 x^{2}}{2}+c$ is the only anti-derivative of $3 x$ because when we differentiate it we get $3 x$. This is a reflection that she accepts an anti-derivative to be of the form $F(x)+c$. This concept image matches her personal definition that an anti-derivative is an integration because when finding the integral the constant of integration is always included. She takes an integral and integration as the same; however, the first is the mathematical object whereas the second is the process.

Some responses to Question 7 had one dimension while others had two dimensions. One-dimensional responses included: $f(x)$ is the integral of $h(x)$ (29 students including S100), $f(x)$ is the anti-derivative of $h(x)$ (24 students including S12, S52, and S41), $f(x)$ is the integration of $h(x)$ (7 subjects including S67), $h(x)$ is the derivative of $f(x)$ (1 subject, S48) and $f(x)$ is the integrant of $h(x)$ (3 subjects). Two-dimensional responses included $h(x)$ is the derivative of $f(x)$ and $f(x)$ is the integral of $h(x)$ (17 students including S42 and S96), $f(x)$ is the integral of $h(x)$ and $h(x)$ is the differential of $f(x)$ (8 students including S25), they are reverse functions (4 students including S59), they are inversely related (4 students) and they are power functions ( 3 students). Categories such as ' $f(x)$ is the opposite of $h(x)$ ' and 'no relationship' fell under individual responses given by the remaining 17 students.

Questionnaire responses of S12 and S42 show consistency of interpretation.

S12: $f(x)$ is the anti-derivative of $h(x)$.

S42: $h(x)$ is the derivative of $f(x)$ and $f(x)$ is the anti-derivative (integration) of $h(x)$.

S12 was asked to explain her response in the interview:

$\mathrm{R}$ : $\quad$ Here you say that $f(x)$ is the anti-derivative of $h(x)$, what do you mean by this?

S12: What I meant is that we got this one $[h(x)]$ when we differentiated and we got this one $[f(x)]$ when we integrated.
In this case she says that there is one anti-derivative because the integration or differentiation had been performed once. This is consistent with her understanding that the number of anti-derivatives of a function depends on the number of times a function had been differentiated.

S42 had earlier described an anti-derivative as the general anti-derivative only. Her response reflects the same earlier interpretation. She had also referred to anti-derivative as integration of a function and she emphasises this conception by enclosing the word integration in brackets after writing anti-derivative. Since in Question 5 the students obtained $h(x)$ from $f(x)$ through the process of differentiation, this shows that the students' understanding of anti-derivative is that the function is an anti-derivative of the other if it yields the function in question when differentiated; this meaning resonates with the definition of an anti-derivative of a function.

\section{RQ3 Assertion: Students failed to apply their concept definitions of anti-derivative and their concept images of integration and differentiation in solving the problems}

The previous sections have shown that students' descriptions associated anti-derivatives with integration and differentiation. When relating functions to their antiderivatives, the two processes also played a major role. This is in alignment with the literature review about antiderivatives. This section presents findings of how students' conceptions may have influenced the way the students solved problems involving integration and differentiation. Before we present the findings of how such problems were solved we begin by giving the findings of how the students related the two processes explicitly in Question 4. The question required the students to show how differentiation and integration are related.

In responding to Question 4, four categories of correct explanations were identified as: reverse (39 students; 33\%), opposite (16 students; 14\%), inverse (12 students; 10\%) and back to original form (8 students; $7 \%$ ). Of the remaining students, $14(12 \%)$ gave individual responses, such as opposite as reverse, opposite as negative and vice-versa, 23 (20\%) gave wrong responses such as 'differentiation is the gradient while integration is the area' (S24) and 5 (4\%) did not respond to the question. Some errors made include confusing the processes of integration and differentiation, the incorrect use of integration and integration operators, and giving the descriptions of how some techniques (e.g. power rule) of the two processes are individually performed without relating the processes. Most of students' descriptions used words such as inverse, reverse and back to original form which resonated with the categories of descriptions of antiderivative in Table 2. Thus students' concept images of antiderivatives and associated processes of integration and differentiation were evoked at different times and did not have some conflicts. This is not only because they were evoked at different times but also because their interpretations were the same when evoked. 
Students' responses to Question 9 follow. This question required students to find (a) $\int \frac{d}{d x}\left(x^{2}\right) d x$ (b) $\int \frac{d}{d x}\left(e^{x^{2}}\right) d x$ and (c) $\frac{d}{d x} \int\left(e^{x^{2}}\right) d x$. The expected answers for the questions were (a) $x^{2}+c$, (b) $e x^{2}+\mathrm{c}$ and (c) $e^{x^{2}}$. Table 3 gives a summary of the type of responses students gave.

Responses to Questions 9(a) and 9(b) were reasonable, but responses to Question 9(c) were problematic because students wanted to break the expressions down into elementary functions that did not exist. This may be because for Questions 9(a) and (b) the differentiation operator was to be applied before the integration operator and the majority of students managed to find the derivative of $e^{x^{2}}$ as $2 x e^{x^{2}}$. A few students wrote the answers to these questions without showing any working. Most students were confined to the manipulations of symbols to get to the results. S42 was among the students who wrote the correct response $\frac{d}{d x} \int\left(e^{x^{2}}\right) d x=e^{x^{2}}$ and it appeared she used the idea that differentiation and integration are reverse processes. The interview with $\mathrm{S} 42$ revealed that this however was not necessarily the case. In her responses to other parts of the questionnaire she had shown that differentiation and integration are opposites of each other.

R: How did you get $e^{x^{2}}$ ?

S42: I integrated.

$\mathrm{R}: \quad$ What did you get as you integrated?

S42: I got $2 x e^{x^{2}}$. I let $u=x^{2}$ and $d u=2 x d x$. So $\int e^{u} d u=e^{u}=e^{x^{2}}$.

$\mathrm{R}$ : But the question does not require you to find $\frac{d}{d x} \int\left(2 x e^{x^{2}}\right) d x$.

S42: Yes but when you differentiate it $2 x e^{x^{2}}$ you get $e^{x^{2}}$.

When S42 finds that the $2 x$ she expects to exist is not available in order to make the manipulation possible, she introduces it so that the expression can be of the form $\int e^{u} d u$, which would be easier to integrate. The student seems to be stuck in procedures and she does not go beyond the manipulation to understanding that if the two processes are opposites or reverses of each other clearly using their operators together depending on which is applied first takes one back to where they started. S42 was further asked some questions that required her to reflect on her actions:

R: In responding to Question 4 you said that differentiation and integration are the opposites of each other, what do you mean by this?

S42: If we take the function $f(x)=3 x$, if we integrate it we get the integral. To go back to the same function we get the derivative and the process is called differentiation.

R: Can you tell me what the result would be here without working, $\int \frac{d}{d x}\left(e^{x^{2}}\right) d x$ ?

S42: $e^{x^{2}}$

TABLE 3: Summary of responses to Question 9.

\begin{tabular}{lccc}
\hline Question & Correct & Incorrect (interviewees) & No response \\
\hline a. & 111 & 6 & 0 \\
b. & 71 & $43(\mathrm{~S} 12, \mathrm{~S} 41, \mathrm{~S} 101)$ & 3 \\
c. & 29 & $86(\mathrm{~S} 12, \mathrm{~S} 41, \mathrm{~S} 101)$ & 2 \\
\hline
\end{tabular}

R: Now with that knowledge what do you think the result here, $\frac{d}{d x} \int\left(e^{x^{2}}\right) d x$, would be?

S42: [Silent]

$\mathrm{R}$ What encouraged you to work out $\int \frac{d}{d x}\left(e^{x^{2}}\right) d x$ ?

S42: Because I knew how to work it out.

R: What about here, $\frac{d}{d x} \int\left(e^{x^{2}}\right) d x$ ?

S42: I just wrote the answer because I did not know how to work it out.

S42 had made connections between the processes of integration and integration. She managed to solve the problems successfully when differentiation was carried out first before integration. But, when integration precedes differentiation she makes the connection between the two processes beyond the algebraic manipulation. This appears to mean that the correct answer was obtained with the understanding that integration and differentiation are opposites of each other but could not give reasons beyond saying she could not work the problem out. In addition, the integrals were written without a constant of integration, which might also be a sign that the order in which the integration and differentiation operators are presented has no significance in terms of the concept they signify or it could be that the student at this moment just forgot to write the constant of integration.

S12 was among the students who said that integration is the opposite of integration; however, her explanation is still clouded by language issues. She wrote:

S12: Because when we differentiate we subtract the constant to complete the differentiation. So since integration is the opposite of differentiation, we add instead of subtract.

S12 persistently uses the words subtract and add to show that the two processes are opposites of each other. Everyday language gets in the way of her explanation. What she means is that when differentiating the constant of integration the result is zero, thus the constant disappears so she uses the everyday word subtract because by subtraction we mean take away and when integrating we add thus we see the constant coming back. Thus she shows a coherent concept image about the way the two processes are related. The only problem is how to explain her actions using proper mathematical language. These findings on language issues are similar to those of Hall (2010). For example, one student said that 'definite integral' is the opposite of 'indefinite integral'. This meaning is also true in everyday life but not in the mathematical context.

Her (S12) written work to Question 10(c) which has the same external structure as Question 9(c) follows:

$$
\text { (c) } \begin{aligned}
\frac{d}{d x} \int e^{x^{3}} d x & =\frac{d}{d x}\left(\frac{e^{x^{3}}}{x^{2}}\right)=\frac{\frac{d}{d x}\left(e^{x^{3}}\right)\left(x^{2}\right)-\left(e^{x^{3}}\right) \frac{d}{d x}\left(x^{2}\right)}{\left(x^{2}\right)^{2}} \\
& =\frac{x^{3} e^{x^{3}} x^{2}-e^{x^{2}} 2 x}{x^{4}}=\frac{x^{6} e^{x^{2}}-e^{x^{2}} 2 x}{x^{4}}
\end{aligned}
$$


R: How did you get your answer here?

S12: Maybe I should have differentiated.

$\mathrm{R}$ : But the integration operator comes before the differentiation one.

S12: But I think I prefer differentiation first before integration.

R: Why do you prefer differentiation first?

S12: Because I think it is going to be a little bit simpler.

S12 divides by the derivative of the power $x^{3}$ as $3 x^{2}$. She then cancels it and leaves $x^{2}$ and applies the quotient rule. None of the algebraic representation and manipulation is correct. This shows that as in the case of S42 she knows that the two processes are opposites of each other (in the mathematical sense) verbally but she cannot use this understanding in solving problems that involve algebraic symbols. Although she says that she would have preferred differentiation before integration her work shows that she cannot perform the procedure for differentiating successfully. Her response to Question 10 (b) further illustrates this point:

$$
\text { (b) } \begin{array}{ll}
\int \frac{d}{d x}\left(e^{x^{3}}\right) \quad f(x)=3 x^{3} \\
=x^{3} e^{x^{2}} \quad g(x)=3 x^{3}+c \\
=\int x^{3} e^{x^{2}} d x \\
=\int x^{2} \cdot x e^{x^{2}} d x \\
\text { Let } u=x^{2} d u=2 x d x \quad d v=x e^{x^{2}} \quad v=e^{x^{2}} \ln 2 x \\
\int x^{2}-x e^{x^{2}}=x^{2} e^{x^{2}} \ln 2 x-\int e^{x^{2}} \ln 2 x d x \\
=x^{2} e^{x^{2}} \ln 2 x
\end{array}
$$

The first step shows that S12 seems to be applying the power rule $\left(\frac{d}{d x}\left(x^{n}\right)=n x^{n-1}\right)$ inappropriately. She takes it to be the power function. She gets $e^{x^{2}}$ by bringing down the $x^{3}$ and reducing its power by one to get $x^{2}$. She seems to have problems in relating the symbolic external structure of the function, the syntax, with the appropriate technique. She takes $e^{u}$ to be of the form $x^{n}$, she tries to apply integration by parts on the wrong expression and this does not help because even here her choice of integration technique does not seem to make sense.

This seems to resonate with the view that symbols in themselves have no meaning until the learner attaches meaning to them (Sengul \& Katranci, 2015). So applying the conception that integration and differentiation are opposites of each other here seems to fail as it seems to have strong association with symbolic manipulation and there is no connection made to the concept. Hence, it is difficult for her to go beyond this stage of manipulation rather than the way the concepts are related in terms of thinking. Thus her concept image of the two processes of differentiation and integration is not coherent. Maharaj (2014) also found that in solving questions on integrals, students got incorrect answers due to lack of proper mental structures. Using the theoretical framework of the reported study this finding could be equated to having an incoherent concept image of integrals as the associated properties and processes to the differentiation and integration concepts in the students' minds could not make sense in solving problems, as indicated by their obtaining incorrect answers.

\section{Discussion}

The study has shown that students gave personal definitions of anti-derivatives that overlapped with the formal definition as reflected in their interviews. These findings are different from those of Hall (2010) as highlighted earlier. While some students accepted the existence of both the general and the particular anti-derivatives, others accepted the existence of the general anti-derivative only. Some questions about antiderivatives were given similar responses but with multiple interpretations. The word anti-derivative was also used synonymously with some other words which did not necessarily carry the same mathematical meaning with it but did have a connection of some sort. These are words such as: integration, integral, inverse and back to original function. Integration is a process used in finding anti-derivatives, an anti-derivative is used synonymously with indefinite integral, the prefix 'anti' in anti-derivative is associated with the word inverse or reverse and since integration and differentiation are reverse processes, it means that applying the process of integration to an integrand gives back the function that one started with.

In some cases the students interpreted the word antiderivative the same. Students persistently showed that a function has one anti-derivative or many in the interviews. Some students failed to use their conceptions of how integration and differentiation are related in solving problems where differentiation and integration operators were paired. The main problem was that students could not go beyond the manipulation of symbols to relating them to the concepts they represent by taking them as reverse processes and sometimes there was no need to carry out any algebraic manipulations but just to write the correct answers. While some of students' conceptions were unique to the context of the study (integral and integration) some were shared across contexts (inverse and back to original function) as in the study of Pino-Fan et al. (2017), which could be a sign that such interpretations were related to the nature of the concept and not necessarily the contexts in which the studies took place. These findings are also similar to those of Maharaj (2014) and Brijlall and Ndlazi (2019), where students got incorrect answers due to failure to recognise the structure or syntax of symbols. We concur with this observation because in our view once the structure of the function as an object is not realised it would be difficult to associate it with the appropriate technique of either differentiating or integrating. With regard to how students interpreted an anti-derivative and the processes of differentiation and integration, there seemed to be no conflicts between students' evoked concept images. As highlighted earlier this is not a conclusion made only due to the fact that the concept images were evoked at 
different times. It is also because when such concept images were evoked they were not at variance with the ones already evoked in the preceding stages.

\section{Implications}

The purpose of teaching is to promote understanding; questionable understanding means questionable teaching. This may be true as in the case of the way Question 5, Question 6, and Question 7 in the reported study are written. The restriction, $n \neq-1$, should have been written in each of those questions so that unnecessary generalisation is not made by students. In class students had been taught that when integrating a function whose power is negative one $(-1)$ the power rule of integration does not apply as the denominator becomes zero, hence the integral becomes $\ln |x|+c$. Such errors created by lack of precision when asking questions by educators may lead to improper understanding of a concept. Meaningful understanding can be achieved if appropriate representation, connections between mathematical ideas and procedural operations are made. Thus the implications to be suggested from this study should be that mathematics teaching needs to consider the semiotics and stress the explicit consideration of the connections between sign, idea and process and the syntaxsemantics relationship. These include: asking students to explain their answers, discussing the meanings of technical terms in class, putting emphasis on the relationship between syntax and semantics, and stressing the association between concept labels (words) and associated experiences and operations. Implementing these ideas has the potential to close the gap between the teachers' (including the mathematical community) and students' interpretation of the same mathematical idea. What remains a challenge for future research is investigating the complex manner in which mathematical knowledge is constructed.

\section{Conclusion}

It is evident from the study that students do sometimes make their own reconstruction of mathematical ideas taught. The mental structures that students have about the concept antiderivative is not fully coherent as a result. There are a number of instances where students failed to solve problems on integration and differentiation even though they had related the two concepts correctly verbally. This shows that making connections between symbols and the concepts they represent is still a challenge that needs further attention.

Although there has been a claim about data triangulation, when dealing with a very large group of students it is not possible to interview each and every one of them because of the enormity of the exercise. Because of this the choice of 10 students for interviews cannot be claimed to be a fair representation of the whole class. If a different group was chosen for interviews the result would perhaps have been slightly different. This is one of the limitations of the reported study.

The second limitation is that of the time that elapsed between administering of questions to students and the conducting of the interviews. The researchers had to go through responses to questions given in order to choose the interviewees. This on its own would also affect the results as through curiosity students might have discussed their responses with their peers and might also have checked their textbooks to see if the answers they had given were correct. While this has been a limitation the interview excerpts do show that students still had problems with solving problems given regardless of time that had elapsed. Thus it could be that some students did not bother to check if they were correct based on the fact that they were not going to be given any marks for the responses they gave for the posed questions.

\section{Acknowledgements}

We would like to whole-heartedly thank Larry Yore and Anthony Essien for their powerful and thoughtful comments in the writing of this article.

\section{Competing interests}

The authors declare that they have no financial or personal relationships that may have inappropriately influenced them in writing this article.

\section{Authors' contributions}

E.K.M. contributed in writing the article, reviewed the literature, collected data and analysed data. All these were done by holding a series of discussions with M.Q. where necessary.

\section{Funding information}

This research received no specific grant from any funding agency in the public, commercial, or not-for-profit sectors.

\section{Data availability statement}

Data sharing is not applicable to this article as no new data were created or analysed in this study.

\section{Disclaimer}

The views and opinions expressed in this article are those of the authors and do not necessarily reflect the official policy or position of any affiliated agency of the authors.

\section{References}

Brijlall, D., \& Ndlazi, N.J. (2019). Analysing engineering students' understanding of integration to propose a genetic decomposition. Journal of Mathematic Behavior 55. https://doi.org/10.1016/j.jmathb.2019.01.006

Ferrer, F.P. (2016). Investigating students' learning difficulties in integral calculus. International Journal of Social Sciences, 2(1), 310-314. https://doi.org/10.20319/ pijss.2016.s21.310324

Grundmeier, T.A., Hansen, J., \& Sousa, E. (2006). An exploration of definition and procedural fluency in integral calculus, PRIMUS, 16(2), 178-191. https://doi org/10.1080/10511970608984145

Haeussler, E.F., Paul, R.S., \& Wood, R.J. (2008). Mathematical analysis for business, economics and the life and social sciences (12th ed.). Boston, MA: Pearson economics and
Prentice Hall.

Hall, W.L. (2010). Students' misconceptions of the language of calculus: Definite and indefinite integrals. In Proceedings of the 13th Annual Conference on Research in Undergraduate Mathematics Education. Raleigh, NC: Mathematical Association of

Jones, S.R. (2013). Understanding the integral: Students' symbolic forms. Journal of Mathematics Behavior, 32(2), 122-141. https://doi.org/10.1016/j.jmathb. 2012.12.004 
Kiat, S.E. (2005). Analysis of students' difficulties in solving integration problems. The Mathematics Educator 9(1), 39-59. Retrieved from http://math.nie.edu.sg/ame/ matheduc/tme/tmeV9 1 1/Seah\%20EK.pdf

Larson, R., \& Edwards, B. (2012). Calculus 1 with pre-calculus: A one year course (3rd edn.). Belmont, CA: Brookes.

Maharaj, A. (2014). An APOS analysis of natural science students' understanding of integration. REDIMAT, 3(1), 54-73. https://doi.org/10.4471/redimat.2014.40

Metaxas, N. (2007). Difficulties on understanding the indefinite integral. In J.H. Woo, H.C. Lew, K.S. Park, \& D.Y. Seo (Eds.), Proceedings of the 31st Conference of the Internationa Group for the Psychology of Mathematics Education (Vol. 3, pp. 265-272). Seoul PME. Retrieved from ftp://ftp.math.ethz.ch/EMIS/proceedings/PME31/3/265.pdf

Moru, E.K., \& Qhobela, M. (2013). Secondary school teachers' pedagogical content knowledge of some common students' errors and misconceptions in sets. African Journal of Research in Mathematics, Science and Technology Education, 17(3) 220-230. https://doi.org/10.1080/10288457.2013.848534

Ndlazi, N.J., \& Brijlall, D. (2019). Exploring engineering students' understanding of techniques of integration. Journal for New Generation Sciences, 16(2), 59-75. techniques of integration. Journal for New Generation Sciences,
Pino-Fan, L., Gordillo, W., Font, V., Larios, V., and Castro, W. F. (2017). The antiderivative understanding by students in the first university courses. In B. Kaur W.K. Ho, T.L. Toh, \& B.H. Choy (Eds.), Proceedings of the 41st Conference of the International Group for the Psychology of Mathematics Education (Vol. 4, pp. 41-48). Singapore: PME.

Seale, C. (1999). The quality of qualitative research. London: Sage. https://doi. org/10.4135/9780857020093

Sengul, S., \& Katranci, Y. (2015). Metacognitive aspects of solving indefinite integration problems. Journal of Social and Behavioral Sciences, 197, 622-629. https://doi. org/10.1016/j.sbspro.2015.07.205

Stewart, J. (2009). Calculus (6th ed.). Belmont, CA: Brooks.

Tall, D.O., \& Vinner, S. (1981). Concept image and concept definition in mathematics with particular reference to limits and continuity. Educational Studies in Mathematics, 12, 151-169. https://doi.org/10.1007/BF00305619

Vinner, S, (1991). The role of definitions in the teaching and learning of mathematics. In D.O. Tall (Ed.), Advanced mathematical thinking (pp. 65-81). Dordrecht: Kluwer Academic Publishers. https://doi.org/10.1007/0-306-47203-1_5 